\title{
EDITORIAL
}

\section{Why a series on imaging in the ERJ?}

\author{
A.A. Bankier*, P.A. Gevenois", Y. Sibille
}

At its annual meeting three years ago, the European Respiratory Society celebrated W. C. RoENTGEN's 100th birthday with a symposium essentially dedicated to thoracic imaging. This symposium gave the pulmonary community the welcomed opportunity to recognize the increasingly important contributions that thoracic imaging provides to both respiratory research and clinical care.

Little more than two decades ago, standard chest radiographs were the only imaging technique routinely available to chest physicians. Then the clinical introduction of computed tomography (CT) in the early 1980s revolutionized thoracic imaging. CT not only evolved to a key modality in the mere diagnosis of respiratory disorders, but also had a profound impact on pretherapeutic planing and post-therapeutic follow-up. Soon thereafter, magnetic resonance imaging (MRI) and digital angiographic techniques broadened the spectrum of diagnostic imaging tools available to the respiratory clinician. Positron Emission Tomography (PET) is now the latest imaging modality introduced into clinical practice and, like the afore-mentioned techniques, this is likely to substantially modify the diagnostic approach to thoracic lesions. Interestingly, standard chest radiography, the "work-horse" in respiratory imaging, has lost nothing of its importance over the last two decades. Indeed, standard chest radiography itself underwent important changes, such as the introduction of new film systems, the subsequent reduction of radiation dose, the increase of image quality, and, finally, constantly expanding digitalization.

The sum of these developments has made of thoracic imaging a cornerstone of respiratory medicine. Simultaneously, the diagnostic armamentarium offered by thoracic imaging nowadays requires highly specialized radiologists to generate reasonable and useful information out of increasingly complex imaging algorithms. Now that the filmless digital radiology department is becoming reality in many hospitals, radiologists also play a key role in processing, transmission, and management of imaging data. In contrast to what some might have expected, these developments have tied intensive new links between pulmonary radiologists

*Dept of Radiology, University Hospital of Vienna, Vienna, Austria, " Dept of Radiology, Hôpital Erasme, Université Libre de Bruxelles, Brussels, Belgium and Dept of Pneumology, Cliniques Universitaires de Mont-Godinne, Université Catholique de Louvain, Belgium

Correspondence: A.A. Bankier, Dept of Radiology, University of Vienna, Waehringer Guertel 18-20, A-1090, Vienna, Austria. Fax: 431404004894 and respiratory clinicians and researchers. Clearly, the complex issues in respiratory medicine call out for a multidisciplinary problem-solving approach.

This series on imaging is the logical consequence of the above mentioned developments. Being a wide information platform for the respiratory physician, the European Respiratory Journal now presents a series of state-of-the-art articles on the most recent and "hot" topics in thoracic imaging. The Editorial Board thereby wishes to emphasize that thoracic imaging is one of our key partners in clinical and experimental respiratory medicine. Contributions from experts and researchers in thoracic imaging will therefore always be well received by the Journal.

The scope of our series on imaging is twofold. On one hand, the latest innovations will be presented. Although these articles will explain some technical principles, they will primarily focus on the potential clinical impact of the new techniques, and discuss how the techniques are likely to change existing diagnostic algorithms. On the other hand, groups of disorders and diagnostic algorithms themselves will be discussed in the light of newly introduced radiological techniques. Among others, small airways diseases, pulmonary embolism, pulmonary infections, and pulmonary emphysema will be analysed. Again, special attention will be given to the clinical application of these modalities. Recognized experts in thoracic imaging will author all articles; a concluding article will summarize the key points of the series and indicate directions for future research and cooperation between thoracic radiologists and both respiratory physicians and surgeons.

Specifically, the following topics will be covered: imaging guided interventions in the thorax; virtual tools for imaging the thorax; small airways disease imaged by computed tomography; strategies for imaging pulmonary embolism; the role of fluorine-deoxy-glucose (FDG)-PET in respiratory oncology; pulmonary ventilation imaged by magnetic resonance; quantitative computed tomography assessment of pulmonary emphysema; imaging of pneumonia; new challenges for research and clinical practice in thoracic imaging.

This series on imaging will present a substantial amount of information relevant to the respiratory physician. It is therefore deeply hoped that this series on imaging will appeal to the readership of the European Respiratory Journal. It is also hoped that the series will further stimulate the ongoing cooperation between thoracic radiologists and respiratory physicians, and tie the existing links between the two disciplines even stronger. 